\title{
Energy Efficient Dynamic Network Configuration in Two-Tier LTE/LTE-A Cellular Networks
}

\author{
Jen-Jee Chen \\ Dept. of Electrical Engineering \\ National University of Tainan \\ Tainan, Taiwan 70005 \\ Email: jjchen@mail.nutn.edu.tw
}

\author{
Chung-Hua $\mathrm{Hu}$ \\ Dept. of Electrical Engineering \\ National University of Tainan \\ Tainan, Taiwan 70005
}

\begin{abstract}
With the increasing demand of broadband wireless communication networks, users have an increasing need of the wireless broadband service coverage and the wireless access quality. To alleviate this problem, several novel techniques are proposed. One of these techniques is femtocells. A femtocell can be used to increase the coverage of wireless broadband service indoors or at hotspots and raise both data transmission rate and access quality. Although the use of femtocells can greatly benefit, deploying large number of femtocells consumes tremendous energy. In order to respond to the problem of global climate change, energy saving is an important issue. This paper discusses how to minimize the energy consumption of femtocells and optimize the energy efficiency of networks while still providing both the same data transmission rate and wireless broadband service coverage. In this work, considering path loss, modulation and coding schemes (MCSs), bit-error-rate (BER) and group mobility, we propose a green handover and dynamic femtocell wake up approach. In default, femtocells are stay in idle mode when no user equipment (UE) connecting to it. A femtocell transits to active mode only when detecting UEs and the efficiency of using the femtocell is better than the macrocell. By this way, not only users can benefit from the femtocells but also femtocells can reduce unnecessary power consumption. Simulation results show that our method performs better than the previous methods in power consumption, energy efficiency and throughput in both 3G/4G wireless communication networks.
\end{abstract}

Keywords-energy-efficiency; LTE/LTE-A; OFDMA; femtocell; green communication networks; handover; heterogeneous networks.

\section{INTRODUCTION}

In wireless communication networks, users connect to peers through base stations (BSs). Since the BS must be always available to provide users wireless access service, the power consumption of BSs is much more than that of user equipments (UEs). Recently, in response to the goal of energy reduction of BSs, the concept of green communication networks [1] is proposed. The authors of [1] presented that the future wireless communication network has to be energy efficient and the benchmark weighs the performance of energy efficiency of the network should be employed. [2][3] investigated the energy efficiency issue of wireless networks and proposed a "Green Radio" solution. In the solution, BSs can turn off their transceivers to achieve power saving when no users are connecting to the BSs for data transmission. However, these studies do not take femtocells into account.

There are several works focusing on the handover issue over the Long-Term Evolution (LTE) macrocell-femtocell heterogeneous networks. Ulvan et al. [4] and Zhang et al. [5] proposed novel handover mechanisms which make handover decisions based on the speeds of UEs and their Quality of Service (QoS) to reduce unnecessary handovers and the handover latency. Since the transmission power of femtocells and macrocells are asymmetry, Moon et al. [6] proposed that the macrocells and femtocells shall be given different signal strength thresholds when determining whether to handoff from the macrocell to the femtocell or from the femtocell to the macrocell. However, it does not take the UE velocity into consideration. Wu et al. [7] proposed to consider both signal strength and the UE velocity in the handover algorithm. However, above studies do not take energy-saving issue into account.

Femtocells can be deployed indoors or at hotspot to increase wireless broadband service coverage and raise data access quality. However, when large number of femtocells exists, the total power consumption of femtocells will be tremendously large. Therefore, a well-designed power saving mechanism is needed. Currently, there are several research works [8][9][10] discussing the green energy issue over the macrocell-femtocell heterogeneous network. Ashraf et al. [8][9] proposed a dynamic energy efficient solution to save the power consumption of femtocells, where a femtocell stays in idle mode in default until it detecting a transmitting UE entering its coverage. Thus, femtocells only have to wake up when serving users. However, this makes a femtocell frequently switch between idle and active modes whenever any transmitting user leaving or arriving at the femtocell, which is still not real power efficient. Chen et al.[10] propose a green handover protocol in two-tier OFDMA macrocellfemtocell networks, where a femtocell only wakes up when a moving UE enters its coverage area and the UE can transmit all the data through the femtocell during the dwell time, otherwise the femtocell keeps idle and the UE remains in macrocell. This causes additional energy consumption and handover cost when the user only has few amounts of data to deliver. To solve above drawbacks, this paper proposes a new energy efficient dynamic network configuration method for $4 \mathrm{G}$ OFDMA macrocell-femtocell heterogeneous networks. In the method, we take path loss, bit-error-rate (BER) and group mobility into consideration. To the best of our knowledge, this paper is the first one to consider these three factors in the energy efficient macrocell-femtocell heterogeneous network. 


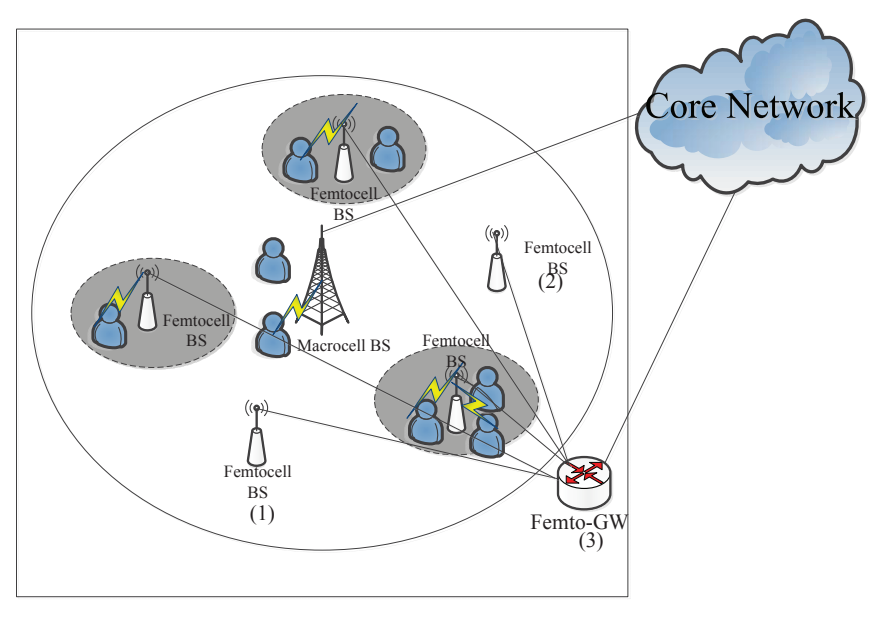

Fig. 1. Macrocell-femtocell heterogeneous network architecture.

This paper considers a dynamic network configuration problem over energy efficient macrocell-femtocell heterogeneous networks. In the heterogeneous network, to maintain service availability, macrocells are always on and thus the power consumption of macrocells is a fixed cost. For UEs, connecting to femtocells is energy conserving compared with connecting to macrocells. However, leaving a femtocell always in active mode will increase unnecessary power consumption of the network. Therefore, there exists a trade-off and the objective of this paper is to maximize the energy efficiency and reduce the total power consumption of the network including macrocells, femtocells and UEs. The contributions of this work are three folds. First, we propose a dynamic network configuration method which performs better energy efficiency and power consumption than previous methods. Second, this work does not have to predict the dwell time of a UE under a certain cell and consider both path loss, BER and group mobility ([8][9][10] are a special case of group size equal to 1), which is more realistic. Third, the performance of our method is evaluated over both $3 \mathrm{G}$ and $4 \mathrm{G}$ systems through simulations. Simulation results show that our proposed method outperforms the previous works on energy efficiency, throughput and total power consumption.

The rest of the paper is organized as follow. Section 2 describes the system model and problem definition. Section 3 presents our proposed energy efficient dynamic network configuration method. Simulation results are shown in Section 4. Section 5 concludes this paper.

\section{System Model and Problem Definition}

\section{A. System Model}

The femtocell[11] is a low power, low cost, and userdeployed equipment. Compared to macrocells, the coverage of femtocells is smaller (e.g. 30-40 $\mathrm{m}$ in diameter). For UEs, femtocells can provide good signal quality because of the short distance. Deploying femtocells can increase coverage for hotspots, improve data transmission rate and offload data traffic for macrocells. Fig. 1 shows the system architecture of a macrocell-femtocell heterogeneous network. The same as macrocells, femtocells work on the licensed band and connect to the operator's core network via DSL broadband backhaul.

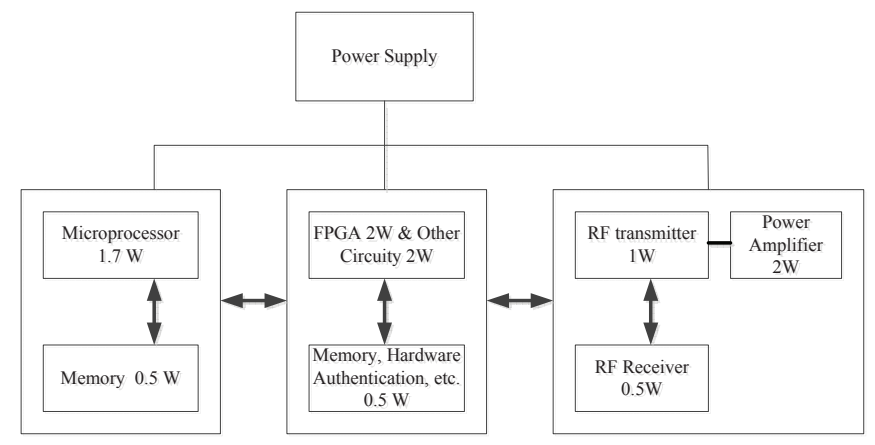

(a)

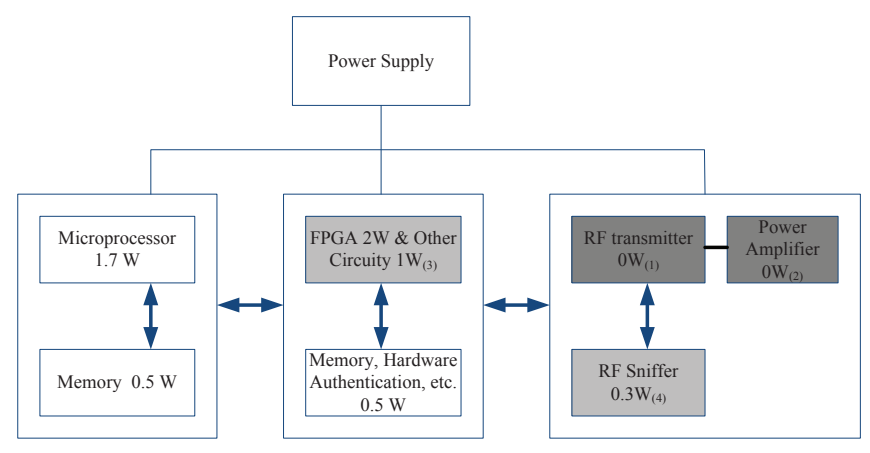

(b)

Fig. 2. Power consumption of femtocell hardware. (a). active mode and (b). idle mode.

To save power, we propose that a femtocell will enter idle mode (Fig. 1 (1)(2)) when no UEs connecting to it; otherwise it will stay in active mode. Femtocells connect to the core network through a femto-gateway (Fig. 1 (3)).

In heterogeneous networks, the femtocell has two spectrum allocation modes [10]: dedicated channel and co-channel modes. In the dedicated channel mode, channels are divided into two parts. One part is only used by femtocells, the other is only used by macrocell. The advantage of the dedicated channel mode is the interference between macrocell and femtocells can be minimized, but this makes low spectrum efficiency. In the co-channel mode, all free channels can be non-simultaneously shared by femtocells and macrocell (but still maintain a part of channels being only used by macrocell), the spectrum efficiency of the co-channel mode is better than that of the dedicated channel mode, but the potential interference between femtocells and macrocell increases. This paper assumes the co-channel mode for our designed method.

Fig. 2 (a) and (b) illustrate the power consumption of hardware modules of idle and active femtocells[10], respectively. The hardware modules are divided into three parts. The first part is random access memory components connect to the microprocessor data handling function. The second part is a field-programmable gate array (FPGA) which implements the data encryption, the hardware authentication and the network time protocol. The third part is the RF transceiver, including separate RF components for the packet transmission and reception, and the RF power amplifier (PA). Fig. 2 (a) shows the power consumption of each module when the femtocell is in the active mode and the total power consumption is $10.2 \mathrm{~W}$. 
TABLE I. THE POWER CONSUMPTION FOR UE AND FEMTOCELL.

\begin{tabular}{|c|c|c|c|}
\cline { 2 - 4 } \multicolumn{1}{c|}{} & Active & Idle & $\begin{array}{c}\text { Power } \\
\text { saving }\end{array}$ \\
\hline femtocell & $10.2 \mathrm{~W}$ & $6 \mathrm{~W}$ & $4.2 \mathrm{~W}$ \\
\hline & $\begin{array}{c}\text { Connect to } \\
\text { macrocell }\end{array}$ & $\begin{array}{c}\text { Connect to } \\
\text { femtocell }\end{array}$ & $\begin{array}{c}\text { Power } \\
\text { saving }\end{array}$ \\
\hline LTE UE & $0.2 \mathrm{~W}$ & $0.0001 \mathrm{~mW}$ & $\approx 0.2 \mathrm{~W}$ \\
\hline $3 \mathrm{G} \mathrm{UE}$ & $1 \mathrm{~W}$ & $\approx 3.2 \mathrm{~mW}$ & $\approx 1 \mathrm{~W}$ \\
\hline
\end{tabular}

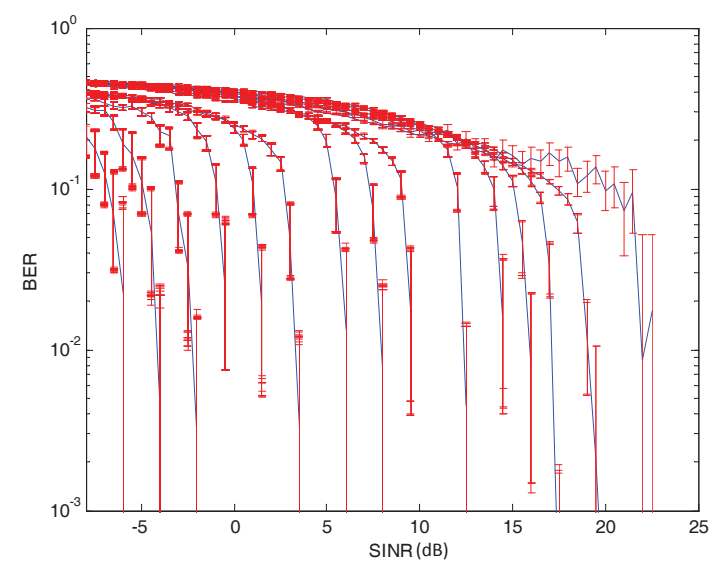

Fig. 3. BER for different CQIs (the 99\% confidence intervals are depicted in red).

On the other hand, Fig. 2 (b) shows the power consumption of each module when the femtocell switches to idle mode. Compared to the active femtocell, an idle femtocell can turn off its RF transceiver (Fig. 2 (b) (1)), power amplifier (Fig. 2 (b) (2)) and miscellaneous hardware components related to nonessential functionalities (Fig. 1 (b) (3)), such as data encryption and hardware authentication. Additionally, since femtocell is in idle mode, a radio sniffer $\left(P_{\text {sniffer }}=0.3 \mathrm{~W}\right)$ (Fig. 2 (b) (4)) module is switched on to measure the power of UE and macrocell. Totally, an idle femtocell consumes $6 \mathrm{~W}$ power. Table I provides the power consumption of data transmission for the UE and the femtocell[10]. For an LTE UE and 3G UE, connecting to the macrocell spends $0.2 \mathrm{~W}$ and $1 \mathrm{~W}$, respectively, but connecting to the femtocell spends an LTE UE and 3G UE only about $0.0001 \mathrm{~mW}$ and $3.2 \mathrm{~mW}$, respectively. Hence the power savings of the LTE UE and 3G UE are about $0.2 \mathrm{~W}$ and $1 \mathrm{~W}$, respectively, if UEs adopt femtocells instead of macrocells. As what has been show in Fig. 2, a femtocell operating in active and idle modes spends $10.2 \mathrm{~W}$ and $6 \mathrm{~W}$, rspectively. if a femtocell keeps staying in idle mode, the power saving is $4.2 \mathrm{~W}$.

LTE uses Channel Quality Indicators (CQIs) to report the current channel condition and each $C Q I=k, k=$ 1..15, has its corresponding Modulation and Coding Scheme (MCS) (denoted by $M C S(C Q I=k)$ ) and rate (denoted by rate $(C Q I=k))[12]$. Furthermore, for different CQI and different BER $(\xi)$, it requires different Signal to Interference plus Noise Ratio (SINR). Fig. 3 shows the required SINR over different $\xi$ for different CQIs[13]. Assume the transmission power of node $i$ is $P_{i}$, the received power of node $j, \tilde{P}(i, j)$, can be written as

$$
\tilde{P}(i, j)=\frac{G_{i} \times G_{j} \times P_{i}}{L_{i, j}},
$$

where $G_{i}$ and $G_{j}$ are the antenna gains of node $i$ and node $j$, respectively, $L(i, j)$ is the path loss. With $\tilde{P}(i, j)$, the received SINR of node $j$ can be derived as follows.

$$
\operatorname{SINR}(i, j)=10 \log _{10} \frac{\tilde{P}(i, j)}{B \times N_{0}+I(i, j)},
$$

where $B$ is the bandwidth, $N_{0}$ is the thermal noise, and $I(i, j)$ is the interference caused by other transmitters which can be presented as $I(i, j)=\Sigma_{l \neq i} \tilde{P}(l, j)$. Note that $\operatorname{SINR}(i, j) \geq$ $\delta\left(C Q I_{i}^{j}, \xi_{i}^{j}\right)$ must be guaranteed, where $\delta\left(C Q I_{i}^{j}, \xi_{i}^{j}\right)$ is the required minimum SINR when $C Q I_{i}^{j}$ and $\xi_{i}^{j}$ are applied by link $(i, j)$.

\section{B. Motivation and Problem Definition}

Ashraf et al. [8][9] proposed to improve energy efficiency by setting femtocells to idle mode when the channel is idle, while switching femtocells to active mode when detecting the signal power of UEs is large than a threshold, thus UEs can always deliver data through femtocells if any femtocell exists. However, femtocells are not always the most energy efficient way to deliver data and additional handover cost is required. On the other hand, Chen et al.[10] proposed a green handover protocol in which each idle femtocell will detect the entry of transmitting UEs and predict UEs' dwell time. If the dwell time is long enough for UEs to transmit all the data via the femtocell, then the femtocell will wake up and UEs handover from the serving macrocell to the femtocell; otherwise, the femtocell will stay idle. Although above method reduces the handover cost, but the energy efficiency is still a problem. This motivates us to propose our solution to maintain an energy efficient communication network with high throughput and low total power consumption. Moreover, we consider path loss, MCSs, BER and group mobility, which are omitted in previous work. The problem is stated as follows.

This paper aims to design an energy efficient dynamic network configuration method for 4G OFDMA macrocellfemtocell heterogeneous networks and the assumptions are as below. (1) There is a group of $N$ UEs moving in the radio access network. There are $M$ femtocells; some are in idle mode (no user connection exists); the others are in active mode (there are UEs connecting to the femtocells). (2) This paper considers path loss, different system types (3G/4G), BER and multiple MCSs. The co-channel allocation mode is used, where all free channels are non-simultaneously shared by femtocells and macrocell. The problem is to determine whether an idle femtocell shall wake up or not when a group of UEs pass by or enter its coverage area and whether the group of UEs shall handover from macrocell to femtocell or not. The objective is to maximize the energy efficiency of overall communication network and maintain both high throughput and low total power consumption. 


\section{ENERGY EFFICIENT DYNAMIC NETWORK CONFIGURATION METHOD}

This section introduces our proposed scheme. Review that we assume a group of $N$ UEs move together. The whole field is covered by the macrocell and $M$ femtocells. Each femtocell is initially open (active) with probability $P$ or sleep (idle) with probability $(1-P)$. For femtocells, the power consumptions are $P_{\text {idle }}^{\text {femto }}$ and $P_{\text {active }}^{\text {femto }}$ when in idle and active modes, respectively. For UEs, the power consumptions of connecting the macrocell and femtocell are $P_{\text {macro }}^{U E}$ and $P_{\text {femto }}^{U E}$, respectively. We denote the available channel data rate of UE $j$ by $R_{\text {macro }}^{j}$ (in bit/s) and $R_{\text {femto }}^{j}$ (in bit/s) when connecting to the macrocell and femtocells, respectively, where $R_{\text {macro }}^{j}$ and $R_{\text {femto }}^{j}$ depend on the channel quality. $R_{\text {macro }}^{j}$ can be obtained by the following equation:

$$
R_{\text {macro }}^{j}=H_{j} \times 12 \times 7 \times F\left(M C S_{k}^{j}\right),
$$

where $H_{j}$ is the amount of physical resource block $(P R B)$ per second reserved to UE $j$ (note that each PRB is composed of 12 subcarriers $\times 7$ symbols), $M C S_{k}^{j}$ is UE $j$ 's available highest channel rate $\mathrm{MCS}$, and $F\left(M C S_{k}^{j}\right)$ represents the symbol data rate of $M C S_{k}^{j}$. The $M C S_{k}^{j}$ that UE $j$ can use, is able to be obtained by the following equation:

$$
\delta\left(C Q I_{i}^{j}=(k+1), \xi_{i}^{j}\right)>\operatorname{SINR}(i, j) \geq \delta\left(C Q I_{i}^{j}=k, \xi_{i}^{j}\right),
$$

where $\operatorname{SINR}(i, j)$ represents the SINR between UE $j$ and the BS $i, \operatorname{SINR}(i, j)$ can be derived by Eq. (2), and $\delta\left(C Q I_{i}^{j}=\right.$ $\left.(k+1), \xi_{i}^{j}\right)$ and $\delta\left(C Q I_{i}^{j}=k, \xi_{i}^{j}\right)$ are the required minimum SINRs of $M C S_{k+1}^{j}$ and $M C S_{k}^{j}$, respectively. So, $M C S_{k}^{j}=$ $M C S_{k}$. For simplicity, no matter UE $j$ is connecting to macrocells or femtocells, we let UE $j$ 's available resource is always $H_{j}$. Although frequencies are spatial reusable, this is out of the scope of this paper and thus we do not discuss this here.

Since femtocells provide high data rate and good channel quality, we assume that UEs can use the most efficient MCS, $M C S_{H}$ when connecting to femtocells. $R_{\text {femto }}^{j}$ can be calculated as follows.

$$
R_{\text {femto }}^{j}=H_{j} \times 12 \times 7 \times F\left(M C S_{H}\right) .
$$

When a group of UEs pass a femtocell, the femtocell will evaluate the energy efficiency to determine whether it shall allow the UEs to handover to it from the serving macrocells. If the femtocell is in idle mode, it will also decide whether to stay in idle mode or transit to active mode according to the evaluation. In the evaluation, we first conduct the energy efficiency of accessing the macrocell by the following equation:

$$
E_{\text {macro }}=\frac{N \times R_{\text {macro }}^{j}}{N \times P_{\text {macro }}^{U E}}=\frac{R_{\text {macro }}^{j}}{P_{\text {macro }}^{U E}},
$$

where we assume the group of $N$ UEs have the same $H_{j}$ and similar channel conditions to simplify the problem. The energy efficiency of accessing the femtocell can be derived by the following equation:

$E_{\text {famto }}= \begin{cases}\frac{N \times R_{\text {femto }}^{j}}{\left(P_{\text {active }}^{\text {femto }}-P_{\text {idle }}^{\text {femto }}\right)+N \times P_{\text {femto }}^{U E}}, & \text { when initially idle } \\ \frac{N \times R_{\text {femto }}^{j}}{N \times P_{\text {femto }}^{U E}}=\frac{R_{\text {femto }}^{j}}{P_{\text {femto }}^{U E}}, & \text { otherwise }\end{cases}$

where $\left(P_{\text {active }}^{\text {femto }}-P_{\text {idle }}^{\text {femto }}\right)$ is the extra power consumption for an idle femtocell switching from idle mode to active mode. If $E_{\text {femto }}>E_{\text {macro }}$, represent that accessing the femtocell is a more energy efficient way than the macrocell for the UEs, i.e., accessing the femtocell, the UEs can deliver more data by spending per joule energy instead of the macrocell; on the contrary, if $E_{\text {femto }} \leq E_{\text {macro }}$, handover to the femtocell is not worthy.

Based on this principle, our method is designed as follows.

1) Once detecting any UE's entry of the femtocell's coverage area, if the femtocell is in idle mode, go to Step 2; otherwise, go to Step 3.

2) Evaluate the energy efficiency of accessing the macrocell and femtocell. If $E_{\text {femto }}>E_{\text {macro }}$, i.e., $\frac{N \times R_{\text {femto }}^{j}}{R_{\text {macro }}^{j}}>\frac{\left(P_{\text {active }}^{\text {femto }}-P_{\text {idle }}^{\text {femto }}\right)+N \times P_{\text {femto }}^{U E}}{P_{\text {macro }}^{U E}}$, the femtocell switches to active mode, waits for the UEs handover requests, and go to Step 4; otherwise, i.e., $E_{\text {femto }} \leq E_{\text {macro }}$, the femtocell keeps staying in idle mode, and go back to step 1 .

$3)$ If $E_{\text {femto }}>E_{\text {macro }}$, i.e., $\frac{R_{\text {femto }}^{j}}{R_{\text {macro }}^{j}}>\frac{P_{\text {femto }}^{U E}}{P_{\text {macro }}^{U E} \text {, the }}$ femtocell determines to allow the UE's handover requests and wait for their requests and go to Step 4; otherwise, i.e., $E_{\text {femto }} \leq E_{\text {macro }}$, the femtocell decides to reject the UE's handover, thus the UEs will continue to stay in macrocell, and go back to step 1.

4) Upon receiving the handover requests of the UEs (here we assume UEs prefer accessing femtocells then macrocells), accept the handover requests and go to Step 5.

5) The femtocell provides radio access service for the UEs.

6) Once the femtocell discovers that there is not radio connection existing, set an OFF timer and switch back to idle mode if there's no connecting request before timeout.

\section{Simulation Results}

To evaluate the proposed energy efficient dynamic network configuration method, we develop a simulation platform by the $\mathrm{C}$ programming language. System parameters are as shown in Table II [14][15][16]. The network size is $1300 \sqrt{2} m \times 1300 \sqrt{2} m$ with one macrocell in the center and $n_{f} \times n_{f}$ femtocells uniformly deployed. Each femtocell is initially active with probability $P$ (with users connecting to it) and idle with probability $(1-P)$ (no user connection). The transmission power of the macrocell and the femtocell is $46 d B_{m}$ and $20 d B_{m}$, respectively. The macrocell supports six MCSs $(Q P S K 1 / 2, Q P S K 3 / 4,16 Q A M 1 / 2,16 Q A M 3 / 4$, $64 Q A M 2 / 3$ and $64 Q A M 3 / 4)$, while the femtocells use $64 Q A M 3 / 4$ to communicate with UEs because of the short 
TABLE II. SIMULATION PARAMETERS.

\begin{tabular}{|c|c|}
\hline \multicolumn{2}{|c|}{ System parameters } \\
\hline Network size & $1300 \sqrt{2} m \times 1300 \sqrt{2} m$ \\
\hline Macrocell support MCS & $\begin{array}{l}Q P S K 1 / 2, Q P S K 3 / 4,16 Q A M 1 / 2, \\
16 Q A M 3 / 4,64 Q A M 2 / 3,64 Q A M 3 / 4\end{array}$ \\
\hline Femtocell coverage range & $55.78 m$ \\
\hline Mobility UEs $(N)$ & $1-10$ \\
\hline Velocity of UEs & $1 \mathrm{~m} / \mathrm{s}-10 \mathrm{~m} / \mathrm{s}$ \\
\hline Deployment Number of femtocell & $n_{f} \times n_{f}\left(n_{f}=1-15\right)$ \\
\hline Thermal noise & $-174 d B_{m} / H_{z}$ \\
\hline \multicolumn{2}{|l|}{ Path loss } \\
\hline \\
\hline \multicolumn{2}{|l|}{$P L_{d B}=15.3+37.6 \log _{10} R$} \\
\hline \multicolumn{2}{|c|}{ Femtocell-femto UE different buildings } \\
\hline \multicolumn{2}{|c|}{$P L_{d B}=\max \left(38.46+20 \log _{10} R, 15.3+37.6 \log _{10} R\right)+0.7 d_{2 D, \text { indoor }}$} \\
\hline Penertration loss & $20 d B$ \\
\hline \multicolumn{2}{|c|}{ Macrocell parameters } \\
\hline Maximum transmit power & $46 d B_{m}$ \\
\hline Maximum antenna gain & $14 d B_{i}$ \\
\hline Noise figure & $5 d B$ \\
\hline \multicolumn{2}{|c|}{ Femtocell parameters } \\
\hline Maximum transmit power & $20 d B_{m}$ \\
\hline Maximum antenna gain & $0 d B_{i}$ \\
\hline Noise figure & $7 d B$ \\
\hline \multicolumn{2}{|c|}{ UE parameters } \\
\hline Maximum transmit power & $23 d B_{m}$ \\
\hline Maximum antenna gain & $0 d B_{i}$ \\
\hline Noise figure & $9 d B$ \\
\hline
\end{tabular}

communication distance between UEs and femtocells. The power consumption of active and idle femtocells is $10.2 \mathrm{~W}$ and $6 \mathrm{~W}$, respectively. The power consumption of $3 \mathrm{G}$ and LTE UEs is as shown in Table I. We simulate a group of $N$ UEs moving in the network. Each simulation result is derived by averaging 1000 experiments. For each UE, it always has data buffered in the network side destined to it. We compare our scheme to Ashraf [8][9] and Chen [10] with the following performance metrics: (1) total amount of extra energy consumption (in $\mathrm{W} \cdot \mathrm{sec}$ ): the total amount of energy consumption minus the fixed consumption of initially active femtocells, (2) throughput (in MByte): the amount of total delivered data from macrocell/femtocells to the UEs, (3) energy efficiency (in bit/joule): the amount of total delivered data from macrocell/femtocells to the UEs divided by the total amount of extra energy consumption, (4) handover frequency (including handovers from macrocell to femtocell and femtocell to macrocell).

\section{A. The Effect of $P$ over $3 G$ and $4 G$ Networks}

Fig. 4 shows the effect of femtocell open probability, $P$, on total amount of extra energy consumption, energy efficiency and average handover frequency over $4 \mathrm{G}$ and $3 \mathrm{G}$ networks. In the simulation, $N=5$, the moving speed of UEs is $1 \mathrm{~m} / \mathrm{s}$ and $n_{f}=10$. Fig. 4(a) and 4(b) show the impact of $P$ on total amount of extra energy consumption over $4 \mathrm{G}$ and $3 \mathrm{G}$ networks, respectively. As we can see from the figures, our method outperforms Ashraf and Chen. Ashraf always selects femtocells if active femtocells exist, while Chen always connects to the macrocell because we assume there is always data in the network for the UEs. Our proposed method intelligently selects an energy-saving way for UEs to deliver data. That is, if femtocells are more energy efficiency than the macrocell, then chooses femtocells; otherwise, choose macrocell. Ashraf performs better in $3 \mathrm{G}$ networks than $4 \mathrm{G}$ networks. This is because for UEs, connecting to femtocells can save $1 W$ power compared to connecting to macrocells in the $3 \mathrm{G}$ network, while the power saving is only $0.2 \mathrm{~W}$ in the
$4 \mathrm{G}$ network, i.e., choosing femtocells in $3 \mathrm{G}$ network is a smart strategy but it may not work in $4 \mathrm{G}$ networks. Fig. 4(c) and 4(d) show the impact of $P$ on the energy efficiency over $4 \mathrm{G}$ and $3 \mathrm{G}$ networks, respectively. Again, our scheme performs better than Ashraf and Chen over both 4G and 3G networks. As $P$ increase, both the energy efficiency of our scheme and Ashraf increase. This is because the femtocells consume less extra energy to serve the group of $N$ UEs. Fig. 4(e) and 4(f) show the impact of $P$ on the average handover frequency over $4 \mathrm{G}$ and $3 \mathrm{G}$ networks, respectively. Chen performs the best because it always chooses to connect to the macrocell. Ashraf performs the worst because it always chooses to connect to the femtocells if femtocells exist. The average handover frequency of our scheme increases as $P$ increases in the $4 \mathrm{G}$ case. This is because the extra energy cost to access a femtocell decreases such that our scheme tends to use femtocells and the average handover frequency increases then.

\section{B. The Effect of $N$ over $4 G$ Networks}

Fig. 5(a) represents the effect of $N$ on the total amount of extra energy consumption per UE over $4 \mathrm{G}$ networks. As we can see from the figure, our scheme performs the best. As $N$ increases, the total amount of extra energy consumption per UE decreases for our method and Ashraf. This is because that more UEs share the extra energy spent by the femtocells when $N$ increases. Fig. 5(b) illustrates the impact of $N$ on the throughput over $4 \mathrm{G}$ networks. As we can see from the figure, the throughput increases when $N$ rises. Our scheme shows slightly better throughput than the other two schemes. Fig. 5(c) shows the impact of $N$ on the energy efficiency over $4 \mathrm{G}$ networks. For all the values of $N$, our scheme always performs the best. Overall, our scheme shows better energy efficiency, total power consumption and throughput than the other two schemes.

\section{CONCLUSiON}

In this paper, an energy efficient dynamic network configuration method is designed for $4 \mathrm{G}$ OFDMA macrocellfemtocell heterogeneous networks. When there are no users connecting to femtocells, they turn off the transceivers and the related circuits and switch to idle mode to save power. Once an idle femtocell detects moving UEs, the dynamic network configuration scheme is executed to determine whether it shall wake up and allow the UEs to handover from macrocell to femtocell or not (for an active femtocell, it also has to decide whether to accept the UEs or not). Our method evaluates the energy efficiency of connecting to the macrocell and the femtocells and makes decision accordingly. To design a general solution, the proposed method takes group mobility, path loss, MCSs, BER and the type of networks into account. Simulation results show that our scheme outperforms previous works in energy efficiency, total power consumption and throughput regardless of $3 \mathrm{G}$ and $4 \mathrm{G}$ systems. In the future, we would like to discuss and evaluate our scheme under the non-saturated traffic condition.

\section{ACKNOWLEDGMENT}

This research is co-sponsored by MOST grants 103-2221E-024-005- and 104-2221-E-024-005-. 


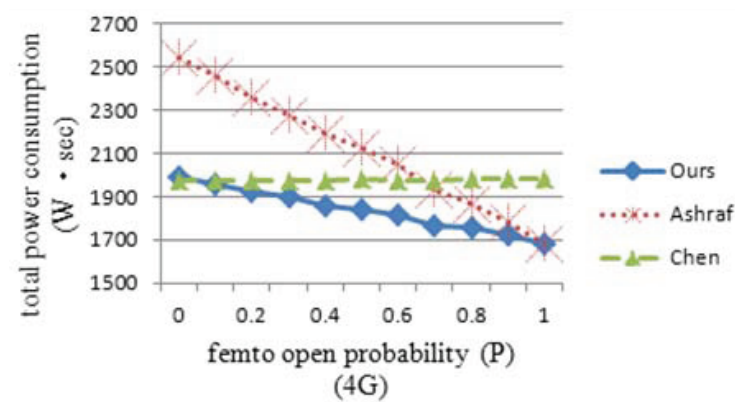

(a)

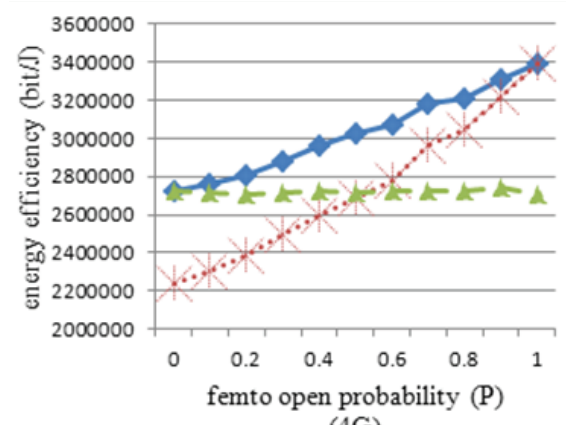

(4G)

(c)

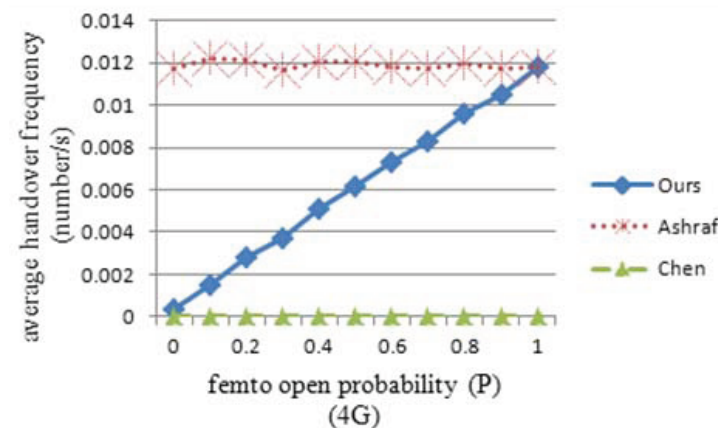

(e)

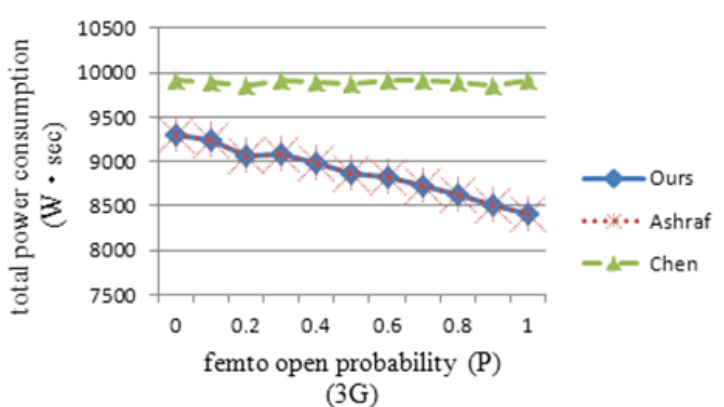

(b)

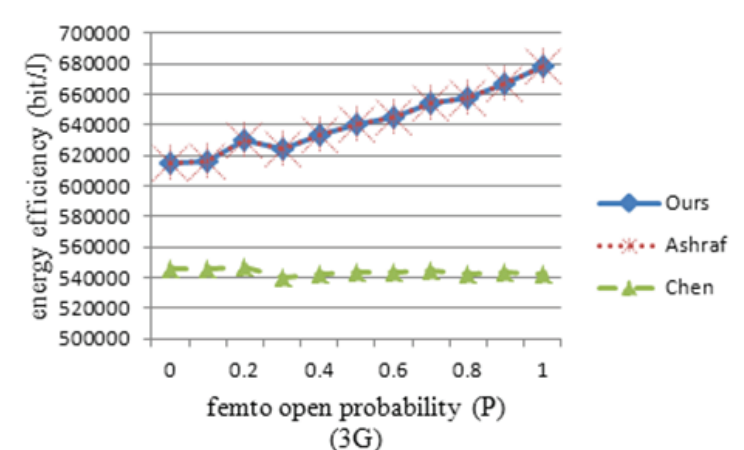

(d)

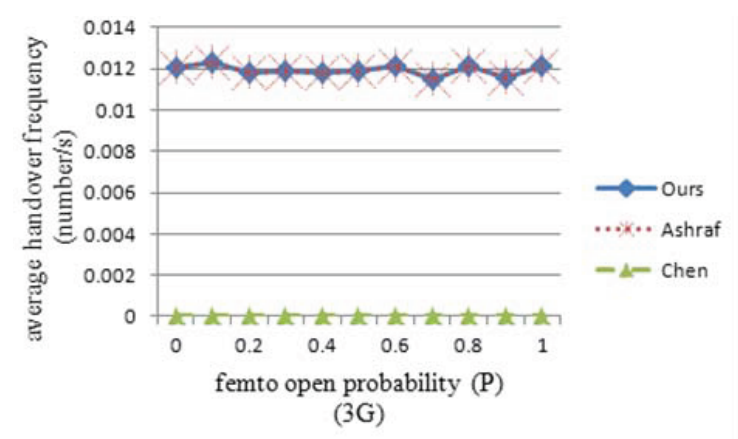

(f)

Fig. 4. The effect of $P$ on (a). extra energy consumption (4G), (b). extra energy consumption (3G), (c). energy efficiency (4G), (d). energy efficiency (3G), (e). average handover frequency (4G) and (f) average handover frequency (3G).

\section{REFERENCES}

[1] K. David and N. Jefferies, "Wireless Visions: A Look to the Future by the Fellows of the WWRF." IEEE Vehicular Technology Magazine, pp. 26-36, Dec. 2012.

[2] T. Chen, Y. Yane, H. Zhang, H. Kim, and K. Horneman, "Network energy saving technologies for green wireless access networks." IEEE Wireless Communications, pp. 30-38, Oct. 2011.

[3] C. Han, T. Harrold, J. Timothy, S. M D. Armour, I. Krikidis, S. Videv, Peter M. Grant, H. Haas, John S. Thompson, I. Ku, C.X.X. Wang, T.A. Le, R.R. Nakhai, J. Zhang, and L. Hanzo, "Green radio: radio techniques to enable energy-efficient wireless networks." IEEE Cоттиnications Magazine, pp. 46-54, May 2011.

[4] A. Ulvan, R. Bestak, and M. Ulvan, "The Study of Handover Procedure in LTE-based Femtocell Network." In Proc. of Wireless and Mobile Networking Conference (WMNC), pp. 1-6, October 2010.

[5] H. Zhang, X. Wen, B. Wang, W. Zheng, and Y. Sun, "A Novel Handover Mechanism between Femtocell and Macrocell for LTE based Networks." In Proc. of International Conference on Communication Software and Networks (ICCSN 2010), pp. 228-231, Feb. 2010.

[6] J. M. Moon and D. H. Cho, "Novel Handoff Decision Algorithm in Hierarchical Macro/Femto-Cell Networks." In Proc. of IEEE Wireless
Communications and Networking Conference (WCNC 2010), pp. 1-6, 2010.

[7] S. Wu, X. Zhang, R. Zheng, Z. Yin, Y. Fang, and D. Yang, "Handover Study Concerning Mobility in the Two-hierarchy Network." In Proc. of IEEE Vehicular Technology Conference (VTC 2009-Spring), pp. 1-5, 2009.

[8] I. Ashraf, L.T.W. Ho, and H. Claussen, "Improving Energy Efficiency of Femtocell Base Stations via User Activity Detection." In Proc. of IEEE Wireless Communications and Networking Conference (WCNC 2010), pp.1-5, April 2010.

[9] H. Claussen, I. Ashraf, and L. T.W. Ho, "Dynamic idle mode procedures for femtocells." Bell Labs Technical Journal, 15(2), pp. 95-116, 2010.

[10] Y. S. Chen and C. Y. Wu, "A Green Handover Protocol in Two-Tier OFDMA Macrocell-Femtocell Network." Mathematical and Computer Modeling (Elsevier), 2012.

[11] V. Chandrasekhar, J. G. Andrews, and A. Gatherer, "Femtocell Networks: A Survey." IEEE Communications Magazine, pp. 59-67, Sep. 2008.

[12] 3GPP TS 36.213 v12.0.0, "E-UTRA; Physical layer procedures." Dec. 2013.

[13] J. Blumenstein, J. Ikuno, J.C. Prokopec, and M. Rupp, "Simulating the long term evolution uplink physical layer.” In Proc. ELMAR, pp. 141- 


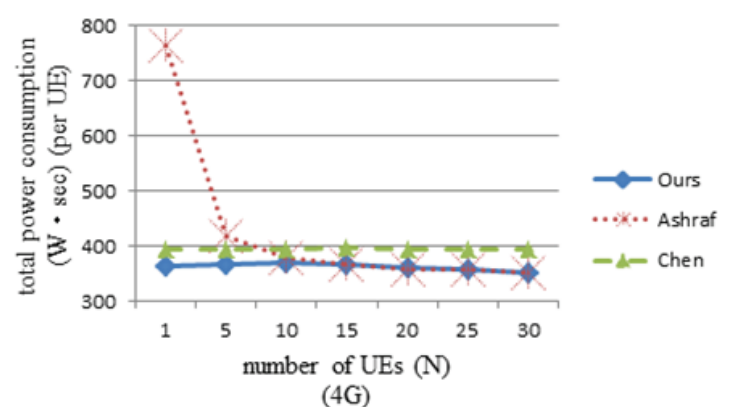

$(4 \mathrm{G})$

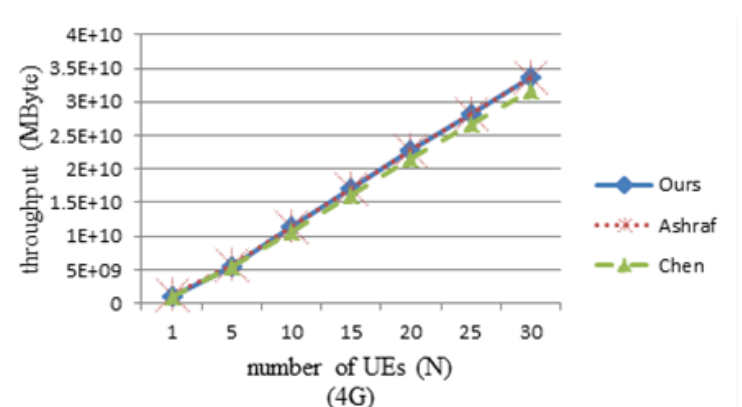

$(4 \mathrm{G})$

(b)

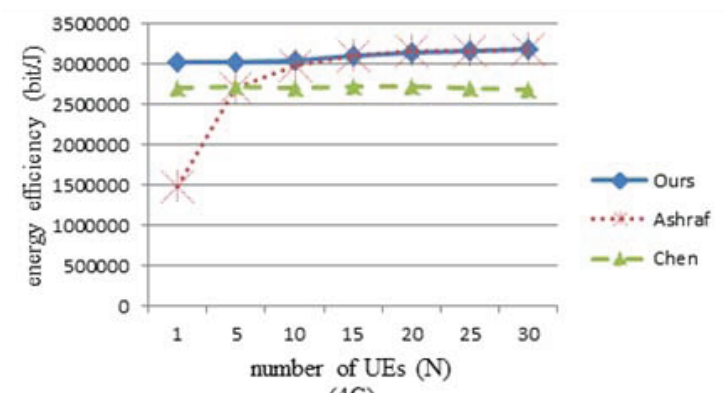

$(4 \mathrm{G})$

(c)

Fig. 5. (a). extra energy consumption V.S. number of UEs $(N)(4 \mathrm{G})$, (b). throughput V.S. number of UEs $(N)$ (4G) and (c). energy efficiency V.S. number of UEs $(N)(4 \mathrm{G})$.

144, Sep. 2011.

[14] S. Sesia, T. Issam, and B. Matthew, "LTE-The UMTS Long Term Evolution." From Theory to Practice, published in 2009, pp. 66.

[15] 3GPP TR 36.814 V9.0.0, "Evolved Universal Terrestrial Radio Access (E-UTRA); Further advancements for E-UTRA physical layer aspects (Release 9)." March 2010.

[16] Z. Zheng, J. Hamalainen, and Y. Yang, "On Uplink Power Control Optimization and Distributed Resource Allocation in Femtocell Networks." In Proc. of IEEE VTC-Spring, pp.1-5, May 2011. 